\title{
CHARACTERIZATION OF AIRCRAFT DYNAMIC WAKE VORTICES AND ATMOSPHERIC TURBULENCE BY COHERENT DOPPLER LIDAR
}

\author{
Songhua $\mathrm{Wu}^{1,2 *}$, Xiaochun $\mathrm{Zhai}^{1}$, Bingyi Liu ${ }^{1}$, Jintao Liu ${ }^{1}$ \\ ${ }^{1}$ Ocean Remote Sensing Institute, Ocean University of China, China, *wush@ouc.edu.cn \\ ${ }^{2}$ Laboratory for Regional Oceanography and Numerical Modeling, Qingdao National Laboratory for \\ Marine Science and Technology, China
}

\begin{abstract}
Field observations for the wake vortices by Coherent Doppler Lidar (CDL) have been carried out at the Beijing Capital International Airport (BCIA) and Tianjin Binhai International Airport (TBIA) to investigate the wake vortices evolution characteristics and the near-ground effect. This paper introduces the dynamic wake vortices and atmospheric turbulence monitoring technique, successfully demonstrating that the CDL can capture the key characteristics of wake vortices in real-time, including wake vortices intensity, spatial-temporal evolution and so forth.
\end{abstract}

\section{INTRODUCTION}

The formation of aerodynamic lift force as a result of aircraft motion in airspace is always accompanied by the formation of a pair of vortices. Strong vortices generated by heavy aircraft present a potential hazard to other aircraft following close behind. In times of increasing air traffic and aircraft dimensions, the wake vortices issue is becoming more important for flight safety, airport capacity, and aircraft design. Several research programs based on simulations, model calculations, wind tunnel and so forth have been carried out [1-3]. For experimental investigations of wake vortices in the atmospheric environment, the Coherent Doppler Lidar (CDL) has been proved to be the most effective and flexible tool along with the developed measuring strategies and methods for processing of raw Lidar data [4-9].

In order to investigate the wake vortices evolution characteristics and the near ground effect, field observation of wake vortices utilizing CDL has been carried out at BCIA and TBIA since 2014. This paper gives a brief analysis of the dynamic wake vortices and atmospheric turbulence characteristics monitoring technique.

\section{METHODOLOGY}

Range Height Indicator (RHI) scanning mode is used with a fixed azimuth angle and varying elevation during the field experiment. As can be seen in Figure 1(a), the color indicates the averaged tangential speed in each detection volume. The center of the pair of positive and negative radial velocity can roughly illustrate the core position of each wake vortex at height of 200 $m$. The Doppler spectrum broadening due to wake vortices is provided in Figure 1(b). To derive wake vortices characterization in real time, the range bins of wake vortices are firstly determined based on Doppler spectrum width.
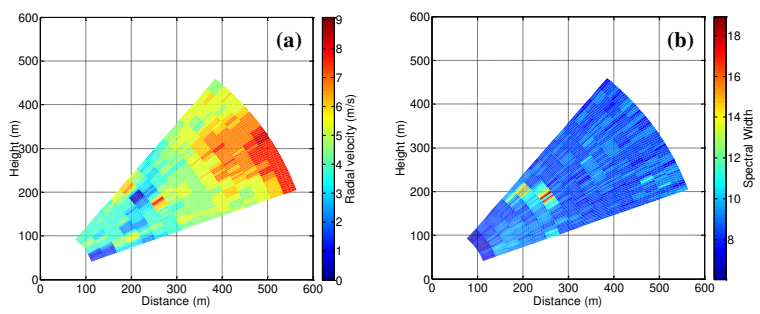

Figure 1 (a) Distribution of the mean of positive and negative velocity along the laser beam (b) Distribution of the spectrum width along the laser line

After the determination of wake vortices range bin, the wake vortices exact location (i.e. the elevation angle of wake vortex) is obtained using the specific distribution of the Doppler spectrum. Figure 2(a) shows an example of the Doppler spectrum versus the velocity and elevation angle at a fixed range which equals to the distance from the Lidar to the left vortex core. The spectrum shown in Figure 2(b) are taken from the data in Figure 2(a) at different elevation angles, labeled with 1,2 , and 3 . Graph 1 shows a spectrum measured outside the vortex area, and graph 2 and 3 show the measurement below and above of the left wake vortex core, respectively. According to the result in Figure 2(b), one can select an appropriate threshold to find two points of the intersection with the spectrum curve to the right 
and to the left of the main spectrum peak, which consists of the velocity envelopes for exact core position determination.
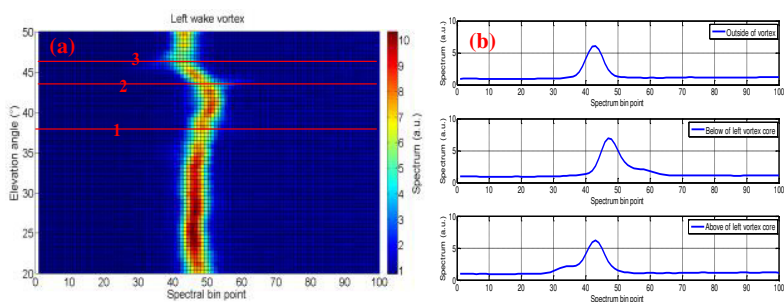

Figure 2 (a) Doppler spectrum measured at different elevation angles and fixed range bin (b) spectrum of (top) outside, (middle) below and (bottom) above the left vortex core.

\section{RESULTS}

\subsection{Wake vortices characteristics evolution}

The velocity envelope distribution with regard to transverse distance near the core areas from descending scan is shown in Figure 3 marked with dotted line. For each radius, a pair of positive and negative velocities can be obtained. The mean value of the velocity pair stands for the velocity along the circle with this radius. In this case, the circulations of these two wake vortices are calculated from the mean value within a distance of $5 \mathrm{~m}$ and $15 \mathrm{~m}$ from the vortices core position, which are $336 \mathrm{~m}^{2} / \mathrm{s}$ and $318 \mathrm{~m}^{2} / \mathrm{s}$, respectively. It is noted that the envelopes include the radial wind velocity and it needs to be derived and then subtracted before the calculation of wake vortices circulation. Figure 4 shows the B744 wake vortices spatial-temporal evolution process on 16:38:07-16:35:51 LT 5 March 2014 at BCIA. Obviously, the wake vortices move away from Lidar because of the crosswind.
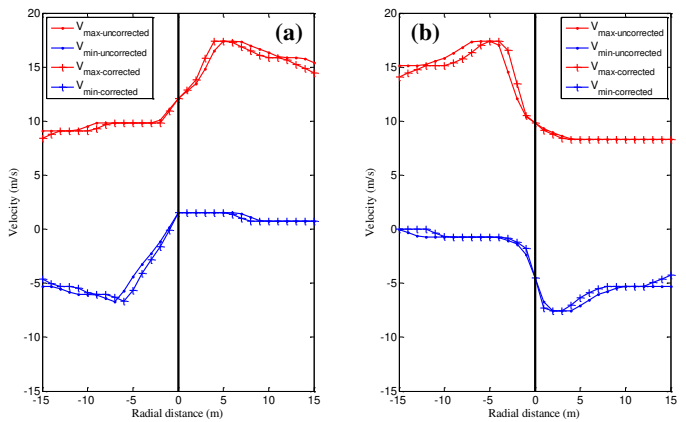

Figure 3 Velocity envelope distribution around the core area before (dotted line) and after correction (plus line) for descending scan. (a) left wake vortex (b) right wake vortex.

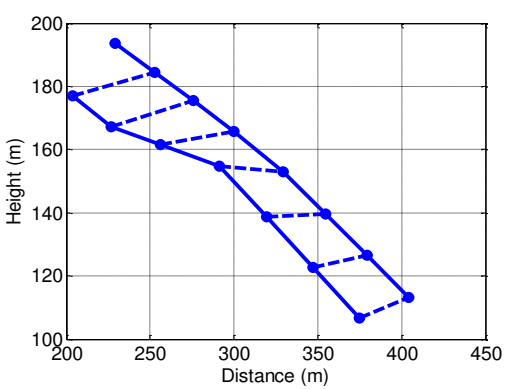

Figure 4 Wake vortex time-spatial evolution process after a B744 flew over during 16:38:07-16:38:51 LT 5 March 2014 at BCIA.

In order to capture the wake vortices with high updating rate, both ascending and descending scans are used alternately to make a reciprocating scanning. However, as the wake vortices usually move downwards, the descending (ascending) Lidar scan results in an expanded (compressed) spatial scale of wake vortices, as a consequence, the value of vortex circulation is overestimated (underestimated) as shown in Figure 5(a). Therefore, the "real" dimension of wake vortices should be firstly corrected to calculate the circulation precisely [5]. Basically, the trajectory and speed of vortices obtained from a series of descending and ascending scans can be estimated to correct the expanded (compressed) wake vortices to obtain its exact dimension and circulation. The results marked with plus line in Figure 3 is the corrected distribution of vortices velocity envelope with transverse distance for descending scan. It can be seen that after the scale of $\mathrm{x}$-axis and interpolation, the corresponding velocity envelope values are less than the uncorrected ones, correcting the overestimate for the expanded wake vortices.

Figure 5(b) shows the wake vortices circulation evolution after correction. Compared to Figure 5(a), the fluctuations of wake vortex circulation value, due to the relative motions of the vortices and laser probing beam, are corrected. Expect for the obvious diminishing trend, it is found that the circulation of left wake vortex (upstream vortex in this case) is always greater than the right wake vortex's (downstream vortex), which is consistent with the fact that the lifetime of upstream vortex is longer than downstream vortex's in the 
crosswind affection.
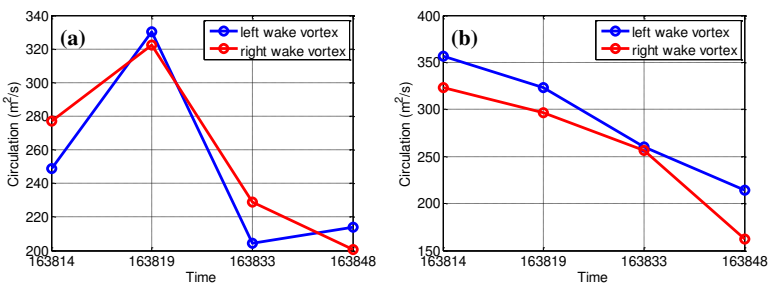

Figure 5. Wake vortex circulation evolution (a) before correction and (a) after correction during 16:38:0716:38:51 LT 5 March 2014 at BCIA.

\subsection{Atmospheric turbulence retrieval using RHI scanning mode}

The spatial statistics of the velocity field are described by the structure function. In many cases, the structure function has a simple description in terms of two parameters: the integral length scale $L_{i}$ and the turbulence energy dissipation rate (TEDR) $\varepsilon$. Before each aircraft overflight, the line-of-sight (LOS) velocity components measured by CDL can be used to calculate the vertical profiles of the crosswind and $\varepsilon$, important parameters for understanding the effect of the atmospheric turbulence on the decay of aircraft wake vortices. The algorithm for extracting high resolution turbulence parameters using longitudinal structure function based on RHI mode has been described in detail by Smalikho et al. [6]

Generally, the values of crosswind velocities at different altitudes $h$ should be calculated as follows,

$$
V_{T}(h)=\frac{1}{N_{a}} \sum_{n} \sum_{l} V_{D}\left(h_{n l}\right) / \cos \varphi_{n},
$$

where the summation is performed satisfying the condition $h-\delta / 2<h_{n l}<h+\delta / 2$, and $N_{a}$ is the number of the values $h_{n l}$ in the averaging layer.

Then the 2D distribution of the mean radial velocity can be estimated as

$$
<V_{D}\left(R_{l}, \varphi_{n}\right)>=V_{T}\left(h_{n l}\right) \cos \varphi_{n},
$$

which is shown in Figure 6(b). The fluctuation of the mean radial velocity is shown in Figure 6(c), according to the equation as

$$
V_{D}^{\prime}\left(R_{l}, \varphi_{n}\right)=V_{D}\left(R_{l}, \varphi_{n}\right)-<V_{D}\left(R_{l}, \varphi_{n}\right)>,
$$

An estimate of the $\varepsilon$ can be obtained from the longitudinal structure function of the wind radial speed as follows,

$\hat{D}_{v}\left(r_{i}, h\right)=\left(N_{s} L\right)^{-1} \sum_{n=1}^{N_{s}} \sum_{h-\delta<h_{k i}<h+\delta}^{L} D_{v}\left(r_{i}, h_{k l i}, n\right)$,
$\hat{D}_{e}\left(r_{i}, h\right)=\left(N_{s} L\right)^{-1} \sum_{n=1}^{N_{s}} \sum_{h-\delta<h_{k l i}<h+\delta}^{L} D_{e}\left(r_{i}, h_{k l i}, n\right)$,

$D_{v}\left(r_{i}, h_{k l i}, n\right)=\left[V_{D}^{\prime}\left(R_{k}+r_{i}, \varphi_{l}, n\right)-V_{D}^{\prime}\left(R_{k}, \varphi_{l}, n\right)\right]^{2}$,

$D_{e}\left(r_{i}, h_{k l i}, n\right)=\left[e\left(R_{k}+r_{i}, \varphi_{l}, n\right)-e\left(R_{k}, \varphi_{l}, n\right)\right]^{2}$,

where $r_{i}=i \Delta R, i=1,2, \ldots, 16, h_{k l i}=\left(R_{k}+r_{i} / 2\right) \sin \varphi_{l}$, $R_{k}=R_{0}+k \Delta R$.

To investigate the influence of turbulence on the aircraft wake vortices, we retrieve the height profiles of $\varepsilon$ from Lidar data measured during 20 scans. In accordance with the results of numerical simulation, this measurement period of $5 \mathrm{~min}$ should be optimal. Figure 7 shows the structure function estimates of turbulence using 20 RHI scans on 17:35:34-17:56:18 18 August 2016 at TBIA with an azimuth angle $\varphi=30^{\circ}$ and height $H=210 \mathrm{~m}$. The $\varepsilon$, standard deviation of the transverse radial velocity $\sigma$ and the integral length scale $L_{i}$ is $2.02 \times 10^{5} \mathrm{~m}^{2} / \mathrm{s}^{3}, 0.21 \mathrm{~m} / \mathrm{s}$ and $281.11 \mathrm{~m}$, respectively. Figure 8 shows the height profiles of $L_{i}, \sigma$ and $\varepsilon$ at corresponding time period.
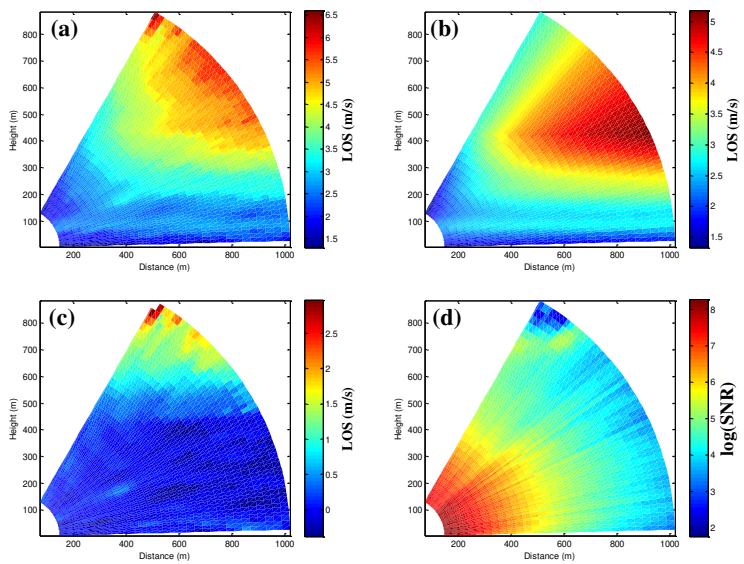

Figure 6 The 2D distribution of (a) radial velocity (b) mean radial velocity $(\boldsymbol{c})$ radial velocity fluctuation $(\boldsymbol{d})$ Signal-to-Noise Ratio (SNR) results using RHI scan mode during 17:35:34-17:56:18 LT August 182016 at Tianjin airport 


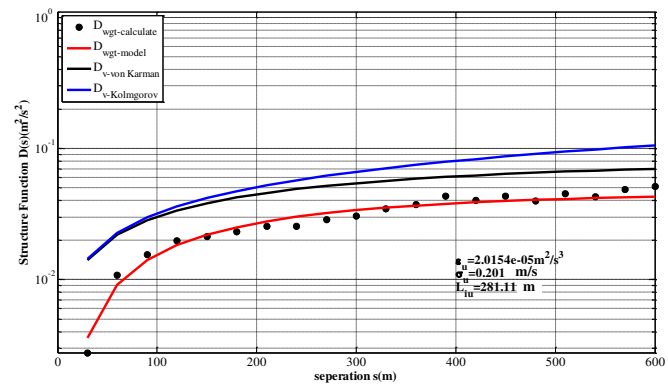

Figure 7. Structure function estimates of turbulence using 20 RHI scans on 17:35:34-17:56:18 LT August 182016 at Tianjin airport with an azimuth angle $\varphi=30^{\circ}$ height $H=210 \mathrm{~m}$.
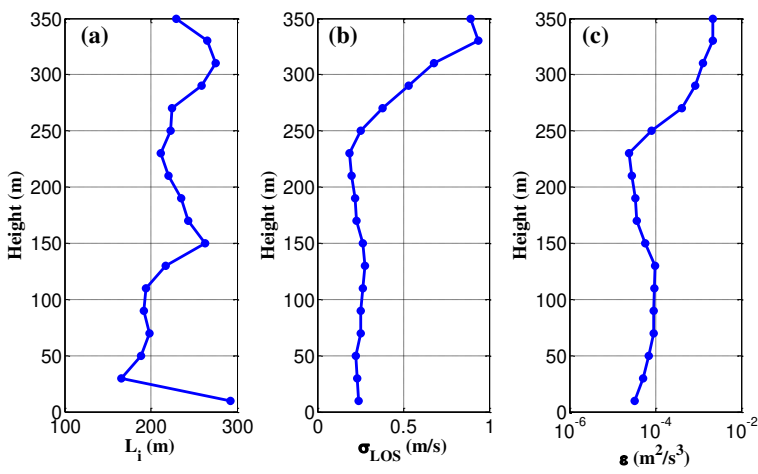

Figure 8 Examples of height profiles of (a) $\varepsilon$, (b) the standard deviation of LOS $\sigma_{L O S}$, (c) the integral length scale $L_{i}$ retrieved by velocity structure function method.

\section{CONCLUSIONS}

In this paper, dynamic wake vortices monitoring algorithm based on the spectral width estimation is introduced. The atmospheric turbulence characteristics are also retrieved using Lidar structure function in RHI mode. Further comparisons and validations are planned in the future. The BCIA and TBIA wake vortices campaigns successfully demonstrate that the concurrent atmospheric turbulence and wake vortices monitoring utilizing coherent Doppler Lidar can describe the key characteristics of wake vortices in real-time, including the wake vortices intensity, spatial-temporal evolution and the interaction with the atmospheric wind and turbulence. The influence of the dynamic state of the atmosphere on the aircraft wake vortices and the near ground effect of wake vortices will be further studied in detail.

\section{ACKNOWLEDGEMENTS}

This work is supported by a joint research program between Boeing-COMAC Sustainable Aviation Technology Center and OUC.

\section{References}

[1] Holz-aring, F., and pfel. 2003: Probabilistic two-phase wake vortex decay and transport model. J. Aircr., 40 (2), 323-331.

[2] Proctor, F. H., Hamilton, D. W., and Han, J. 2000: Wake vortex transport and decay in ground effect: vortex linking with the ground.

[3] Gerz, T., Holzäpfel, F., and Darracq, D. 2002: Commercial aircraft wake vortices. Progress Aerosp. Sci., 38 (3), 181-208.

[4] Köpp, F., Rahm, S., and Smalikho, I. (2004). Characterization of Aircraft Wake Vortices by 2- $\mu$ m Pulsed Doppler Lidar. J. Atmos. Oceanic. Technol., 21 (2), 194-206.

[5] Wu, S., Liu, B., and Liu, J. 2016: Aircraft Wake Vortex Measurement with Coherent Doppler Lidar. In EPJ Web of Conferences (Vol. 119, p. 14008). EDP Sciences.

[6] Smalikho, I. N., and Rahm, S. 2010: Lidar investigations of the effects of wind and atmospheric turbulence on an aircraft wake vortex. Atmos. Oceanic Opt., 23 (2), 137-146.

[7] Holzäpfel, F., Holzäpfel, F., Stephan, A., Stephan, A., Heel, T., Heel, T., ... and Körner, S. 2016: Enhanced wake vortex decay in ground proximity triggered by plate lines. Aircr. Eng. Aerosp. Technol., 88 (2), 206-214.

[8] Smalikho, I. N., and Banakh, V. A. 2015: Estimation of aircraft wake vortex parameters from data measured with a $1.5-\mu \mathrm{m}$ coherent Doppler lidar. Opt. Lett., 40 (14), 3408-3411.

[9] Smalikho, I. N., Banakh, V. A., Holzäpfel, F., and Rahm, S. 2015: Method of radial velocities for the estimation of aircraft wake vortex parameters from data measured by coherent Doppler lidar. Opt. Exp., 23 (19), A1194A1207. 